\title{
Article \\ ZDHHC19 Is Dispensable for Spermatogenesis, but Is Essential for Sperm Functions in Mice
}

\author{
Shuai Wang ${ }^{1}$, Hongjie Qiao ${ }^{1}$, Pengxiang Wang ${ }^{2}$, Yuan Wang ${ }^{3, *}$ and Danian Qin ${ }^{1, *}$ \\ 1 Department of Physiology, Shantou University Medical College, Shantou 515041, China; \\ wangshuai2509@126.com (S.W.); 13hjqiao@stu.edu.cn (H.Q.) \\ 2 Shanghai Key Laboratory of Regulatory Biology, Institute of Biomedical Sciences and School of Life Sciences, \\ East China Normal University, Shanghai 200241, China; wangpengxiang1900@163.com \\ 3 Department of Animal Sciences, College of Agriculture and Natural Resources, Michigan State University, \\ East Lansing, MI 48824, USA \\ * Correspondence: wangyu81@msu.edu (Y.W.); dnqin@stu.edu.cn (D.Q.)
}

Citation: Wang, S.; Qiao, H.; Wang, P.; Wang, Y.; Qin, D. ZDHHC19 Is Dispensable for Spermatogenesis, but Is Essential for Sperm Functions in Mice. Int. J. Mol. Sci. 2021, 22, 8894. https://doi.org/10.3390/ijms22168894

Academic Editor: Youngsok Choi

Received: 22 July 2021

Accepted: 16 August 2021

Published: 18 August 2021

Publisher's Note: MDPI stays neutral with regard to jurisdictional claims in published maps and institutional affiliations.

Copyright: (c) 2021 by the authors. Licensee MDPI, Basel, Switzerland. This article is an open access article distributed under the terms and conditions of the Creative Commons Attribution (CC BY) license (https:/ / creativecommons.org/licenses/by/ $4.0 /)$.

\begin{abstract}
Spermatogenesis is a complicated process involving mitotically proliferating spermatogonial cells, meiotically dividing spermatocytes, and spermatid going through maturation into spermatozoa. The post-translational modifications of proteins play important roles in this biological process. S-palmitoylation is one type of protein modifications catalyzed by zinc finger Asp-HisHis-Cys (ZDHHC)-family palmitoyl $S$-acyltransferases. There are 23 mammalian ZDHHCs that have been identified in mouse. Among them, Zdhhc19 is highly expressed in adult testis. However, the in vivo function of $Z d h h c 19$ in mouse spermatogenesis and fertility remains unknown. In this study, we knocked out the Zdhhc19 gene by generating a 2609 bp deletion from exon 3 to exon 6 in mice. No differences were found in testis morphology and testis/body weight ratios upon Zdhhc19 deletion. Spermatogenesis was not disrupted in Zdhhc19 knockout mice, in which properly developed TRA98+ germ cells, SYCP3+ spermatocytes, and TNP1+ spermatids/spermatozoa were detected in seminiferous tubules. Nevertheless, Zdhhc19 knockout mice were male infertile. Zdhhc19 deficient spermatozoa exhibited multiple defects including abnormal morphology of sperm tails and heads, decreased motility, and disturbed acrosome reaction. All of these led to the inability of Zdhhc19 mutant sperm to fertilize oocytes in IVF assays. Taken together, our results support the fact that Zdhhc19 is a testis enriched gene dispensable for spermatogenesis, but is essential for sperm functions in mice.
\end{abstract}

Keywords: Zdhhc19; spermatogenesis; fertility; sperm motility; acrosome reaction

\section{Introduction}

Spermatogenesis is a complex developmental process, during which spermatogonial stem cells (SSCs) undergo self-renewal and differentiation into spermatocytes and then generate haploid spermatids through two successive meiosis. This highly orchestrated process is controlled by intricate molecular programs including translational and posttranslational regulations. Disturbing spermatogenesis will lead to male infertility [1].

Multiple protein post-translational modifications (PTMs) occur in germ cells during spermatogenesis, such as acetylation [2,3], crotonylation [4], glycosylation [5-7], phosphorylation [8-10], SUMOylation [11], and ubiquitination [12]. Many of these PTMs have been identified to play important roles in sustaining mammalian spermatogenesis and male fertility $[13,14]$. For example, acetylated $\alpha$-TUBULIN is reduced in individuals with poor sperm motility [15]. Chromodomain protein CDYL regulates histone Crotonylation in spermatogenesis. Cdyl transgenic mice manifest dysregulation of histone lysine crotonylation and reduction of male fertility with a decreased epididymal sperm count and sperm cell motility [16]. $\alpha$-mannosidase IIx (MX) is an enzyme involved in $N$-glycan synthesis of proteins. Upon $M X$ gene knockout, mice demonstrate male infertility due to reduced 
GlcNAc-terminated complex type N-glycans [17]. Src homology domain tyrosine phosphatase 2 (SHP2) is a tyrosine phosphatase, depletion of Shp2 in germ cell causes meiotic spermatocytes to die and leads to sterility in mice [18]. Ubiquitination-deficient mutations in P-element Induced Wimpy Testis (PIWI), a key protein for germ cell development, cause male infertility by impairing histone-to-protamine exchange during spermiogenesis [19]. All these studies thus support that proper PTMs are required for maintaining spermatogenesis and male fertility in mice.

Palmitoylation is one type of PTMs, in which proteins are modified with palmitic acids (sixteen-carbon saturated fatty acid) [20,21]. Palmitoylation can occur through $\mathrm{N}$ palmitoylation, $O$-palmitoylation, and S-palmitoylation. S-palmitoylation is the thioesterification that occurs on an internal cysteine residue and is more often found in palmitoylated proteins [22]. S-palmitoylation is a reversible post-translational modification catalyzed by zinc finger Asp-His-His-Cys (ZDHHC)-family palmitoyl S-acyltransferases. By contrast, protein $N$-palmitoylation is essentially irreversible [22]. To date, 23 mammalian ZDHHCs have been identified $[23,24]$. Most ZDHHC proteins are localized to the Golgi and Endoplasmic Reticulum (ER) apparatus [25,26]; however, ZDHHC5, ZDHHC20, and ZDHHC21 are commonly localized to the cell membrane [27]. S-Palmitoylation plays a crucial role in cell signaling, protein localization, and protein-protein interactions [20,21,28,29].

Ohno et al. investigated the ZDHHCs genes expression patterns in human by RT-PCR, and classified ZDHHC genes into roughly three groups according to their tissue-specific expression patterns: highly ubiquitous, nearly ubiquitous, and tissue-specific [27]. The highly ubiquitous and nearly ubiquitous groups each include most of ZDHHC genes (ZDHHC4, 5, 7, 8, 10, 12, 13, 17, and 22; and ZDHHC1, 3, 6, 9, 14, 16, 18, and 21, respectively), indicating that multiple ZDHHC proteins are co-expressed in the same cell. Interestingly, a small number of ZDHHC genes are tissue-specific, including ZDHHC2, 11, 15, 19, and 20. Zdhhc11 and Zdhhc19 mRNAs were unique to testis [27]. We therefore decided to investigate the functions of the Zdhhc19 gene in spermatogenesis by generating Zdhhc19 knockout mice with the CRISPR/Cas9 technology. Male mice lacking Zdhhc19 expression had normal spermatogenesis. However, their fertility was drastically affected. Anomalies were observed in Zdhhc19 deficient sperm, both at the structural and functional levels.

\section{Results}

\subsection{Zdhhc19 mRNA Is Highly Expressed in Testis}

To investigate the role of ZDHHC19 in mouse testis, we first examined Zdhhc19 transcript levels in multiple tissues from adult mice by qPCR. Our results showed that Zdhhc19 mRNA was abundantly expressed in testes, but not detected in other tissues (Figure $1 \mathrm{~A}, \mathrm{C}$ ). Because $\mathrm{Kit}^{\mathrm{w} / \mathrm{wv}}$ mice lack of endogenous spermatogenesis, only supporting cells (e.g., Sertoli cells, Leydig cells, vascular endothelial cells, and fibroblast, etc.) are present in adult mouse testes. We thus collected testes from Kit $t^{t w / w v}$ mice to exclude the possibility that ZDHHC19 is expressed in testicle somatic cells. Indeed, no visible Zdhhc19 transcripts were detected from $\mathrm{Kit}^{w / w v}$ testes (Figure 1A,C). In addition, we found gradually increased Zdhhc19 mRNA expression in testes at postnatal 1-, 2-, 3-, 4-, and 5-week, with the highest level at 5-week (Figure 1B). As meiosis starts in mice around postnatal day 8 , these data suggest a potential role of ZDHHC19 in germ cell development at the postmeiosis stage.

\subsection{Generation of Zdhhc19 Knockout (KO) Mice}

To analyze the physiological functions of $Z d h h c 19$ gene, we utilized the CRISPR/Cas9 technique to establish the Zdhhc19 knockout (KO) mouse model. Homozygous mutant mice were generated and validated by PCR and Sanger sequencing, containing a $2609 \mathrm{bp}$ deletion from exon 3 to 6, which led to frame-shift mutations of $Z d h h c 19$ gene (Figure 2A,B). In addition, qPCR analysis demonstrated that Zdhhc19 mRNA was hardly detected in the testes of Zdhhc19 KO mice (Figure 2C), suggesting that Zdhhc19 gene was successfully 
knocked out. No obvious developmental abnormalities were observed in the Zdhhc19-/-

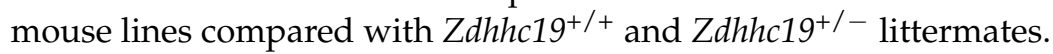

A

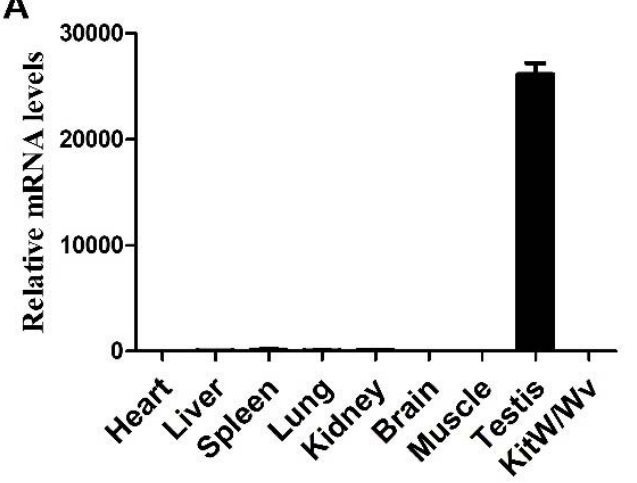

B

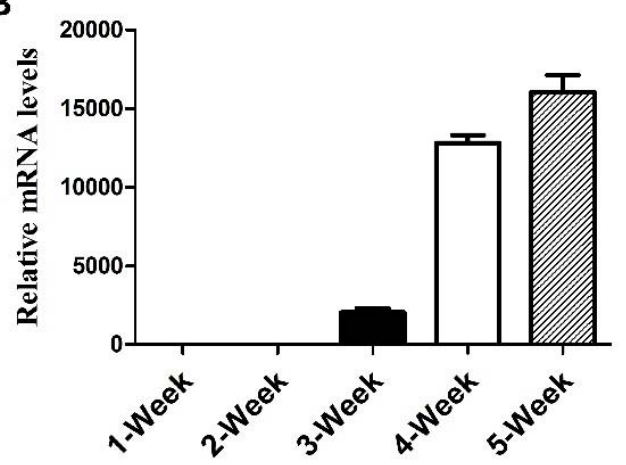

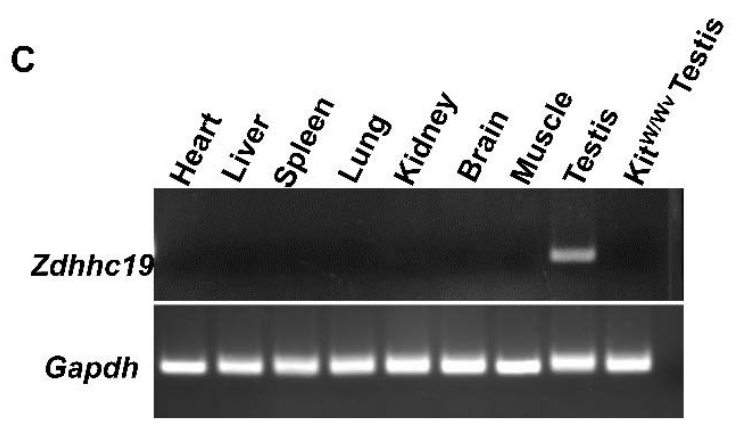

Figure 1. Zdhhc19 is highly expressed in testicular germ cells but not in other tissues. (A) qPCR analyses of Zdhhc19 mRNA expression levels in eight different organs from wildtype adult mice and Sertoli cells (Kit ${ }^{W / W v}$ testis). (B) qPCR analyses of Zdhhc19 mRNA expression levels in developing testes at 1-, 2-, 3-, 4-, and 5-week. (A,B) Zdhhc19 mRNA expression level in heart (A) and 1-Week (B) were used as baseline control, Gapdh served as the cDNA loading control. Data are presented as mean \pm SEM. (C) RT-PCR analysis showed that Zdhhc19 mRNA was only detectable in the testis. No signal was detected in the other eight tissues.

\subsection{Zdhhc19 Deletion Does Not Affect Spermatogenesis}

The deletion of the Zdhhc19 gene could offer an opportunity to understand the functional contribution of $Z d h h c 19$ to mouse spermatogenesis and male fertility. We thus investigated the requirement for Zdhhc19 in mouse spermatogenesis using this Zdhhc19 knockout mouse model.

We dissected the testes of control and $\mathrm{KO}$ mice at $56 \mathrm{dpp}$, and found the testis in $\mathrm{KO}$ mice was morphologically normal (Figure $3 \mathrm{~A}$ ); the testicular size and testes/body weight ratio of $\mathrm{KO}$ mice were comparable to their littermate controls at $42 \mathrm{dpp}$ (Figure $3 \mathrm{~A}, \mathrm{~B}$ ). Postnatal germ cell development in control and $\mathrm{KO}$ mice were then examined with immunohistofluorescence (IHF). SYCP3 is a spermatocyte-specific marker [30], and its signals were comparable in $\mathrm{KO}$ and control mice at $42 \mathrm{dpp}$ (Figure $3 \mathrm{C}$ ), indicating a normal spermatocyte development. TRA98 is a pan-germ cell marker [31], and its staining revealed normal meiosis and haploid germ cell development in $\mathrm{KO}$ mice at $42 \mathrm{dpp}$ (Figure 3C). TNP1, a protein that only marks haploid germ cells [32], was also readily detected in testis sections from $Z d h h c 19 \mathrm{KO}$ mice, indicating the existence of spermatids and spermatozoa in the inner adluminal compartments of seminiferous tubules, similar to those in control littermates (Figure 3D).We further analyzed the epididymides and found no difference in the numbers of spermatozoa in Zdhhc19 KO mice compared to controls (Figure 3E). Finally, we evaluated the impact of Zdhhc19 deletion on male fertility using a breeding test. We found that the breeding performance of Zdhhc19 KO males were significantly reduced. Breeding of three $Z d h h c 19 \mathrm{KO}$ males with wild-type females over a three-month 
period yielded no offspring (Figure 3F). By contrast, wildtype controls gave an average approximately six pups per litter (Figure 3F). In summary, we conclude that Zdhhc19 is dispensable for spermatogenesis, but is essential for sustaining male fertility in mice.

A

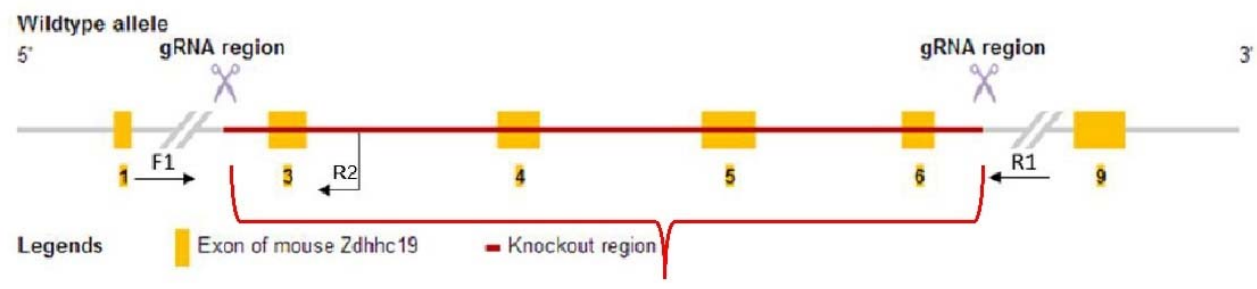

CAACAGTCTAGCTACAGAAAAAGAAATGCGCAGCCATAGA-del 2609 bp-CCACACCAAGAGAGATCAGTCTAGGGCTGTTTAGTTTAGA

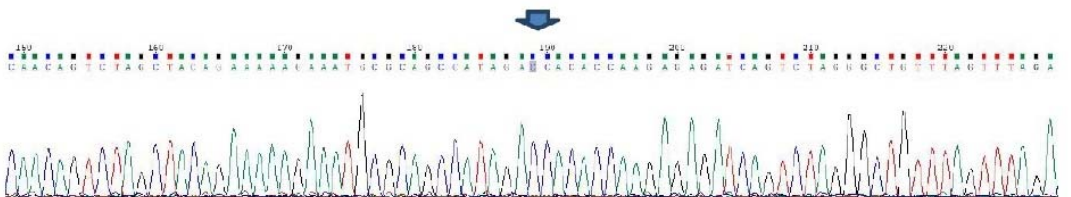

B
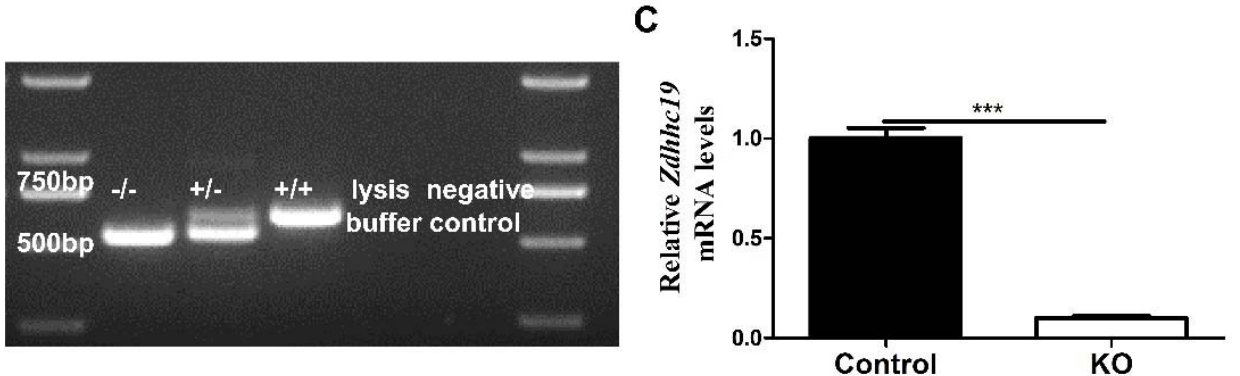

Figure 2. CRISPR/Cas9 strategy to generate Zdhhc19 KO mice. (A) targeting scheme of Zdhhc19 KO mice. Yellow boxes represent exons of the Zdhhc19 gene on mouse chromosome 16, and black arrows (F1, F2 and R1) indicate the site of designed primers for genotyping. Red line indicates the target regions of sgRNAs. Exon 3-6 was deleted upon the injection of the mixtures of Cas9 and Zdhhc19 sgRNA into zygotes. (B) PCR of wildtype (+/+), heterozygous (+/-), and homozygous $(-/-)$. A 536bp band was amplified from the Zdhhc19 KO allele using F1 and R1 primers, and a 617bp band was amplified from the wildtype allele using F1 and R2 primers. (C) Zdhhc19 mRNA expression levels in control and Zdhhc19 KO adult testes were examined by RT-qPCR. Data are presented as mean \pm SEM. ${ }^{* * *} p<0.001$.

\subsection{Knockout of Zdhhc19 Gene Leads to Male Infertility by Affecting Sperm Motility}

Spermatozoa must undergo functional maturation in the epididymis after leaving testis before they can competently interact with oocytes [33]. This process includes the acquisition of sperm motility and the potential to go through the sperm capacitation and acrosome reaction. To understand the underlying causes of male infertility of Zdhhc19 KO mice, we assessed the motility of sperm isolated from the epididymis by CASA. As shown in Figure 4, the sperm motility that was measured by main motility parameters of total motility (Figure 4A) and curvilinear velocity (VCL) (Figure 4B) were significantly decreased upon Zdhhc19 deletion. However, other velocity parameters including straight line velocity (VSL) and average path velocity (VAP) were comparable in $\mathrm{KO}$ and control sperm (Figure 4B). In addition, amplitude of lateral head displacement (ALH) and beat/cross frequency (BCF) were both significantly reduced in Zdhhc19 $\mathrm{KO}$ mice (Figure 4C,D). 
A

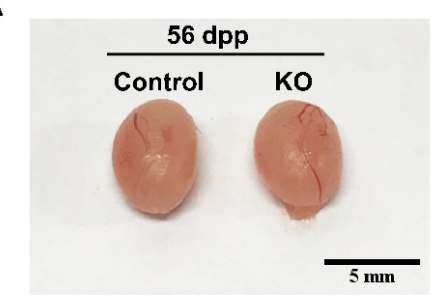

C

C SYCP3
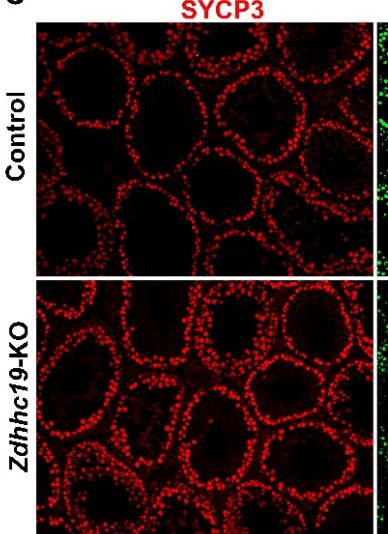

D

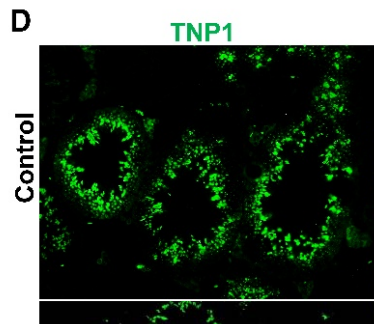

TNP1/DAP
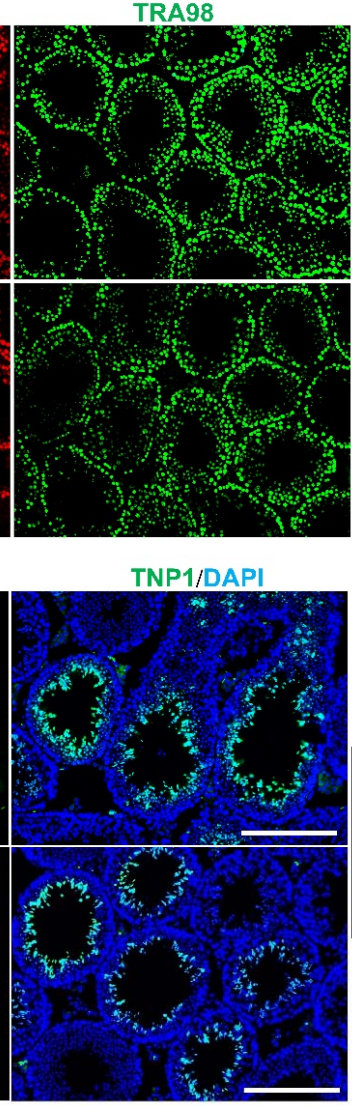

E DAPI

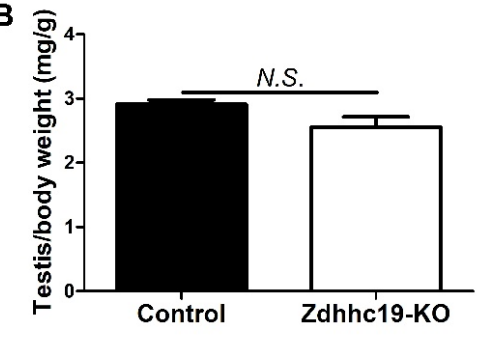

Control
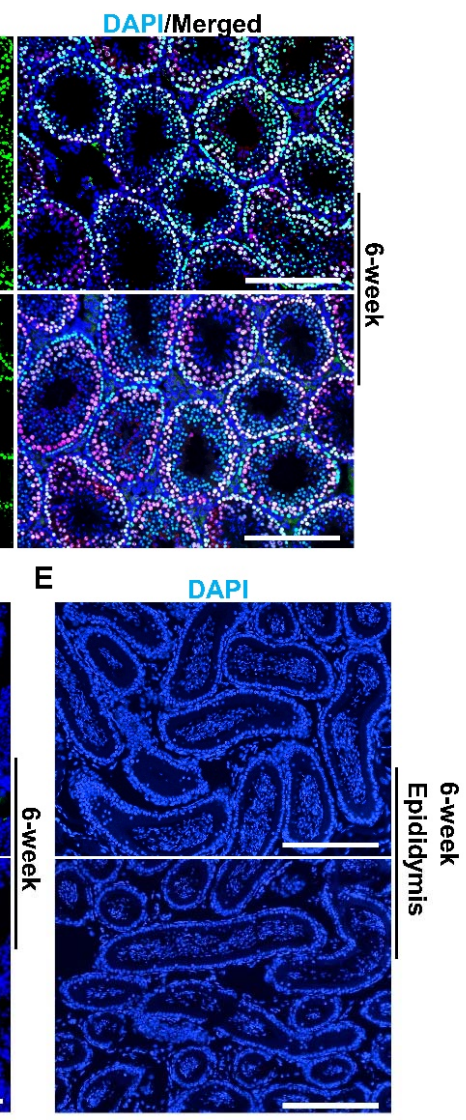

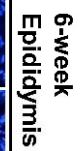

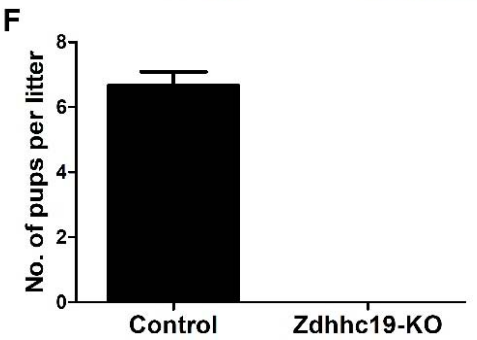

Figure 3. Zdhhc19 deletion does not affect spermatogenesis, but leads to male infertility in mice. (A) Gross morphology of testes from control and KO mice at $56 \mathrm{dpp}$. (B) Averaged testis/body weight ratio was calculated from 4 control and $3 \mathrm{Zdhhc19}$ KO littermates at $42 \mathrm{dpp}$. Data are presented as mean \pm SEM, N.S.: no significance. (C) The development of pan-germ cells (by TRA98 staining) and spermatocytes (by SYCP3 staining) was examined by IHF on $\mathrm{KO}$ and control testis sections from 6-week mice. (D) The elongated spermatids were examined by IHF with a TNP1 antibody, counterstained with DAPI on $\mathrm{KO}$ and control testis sections from 6-week mice. (E) DAPI stained sections of epididymides from control and KO mice at 6-week. (C-E) Scale bars, $200 \mu \mathrm{m}$. (F) Average pups' numbers per litter were calculated based on 6 litters from 3 control and $3 \mathrm{KO}$ male mice, each of which was separately bred with 2 wildtype females for at least 2 months. Data are presented as mean \pm SEM. 
A

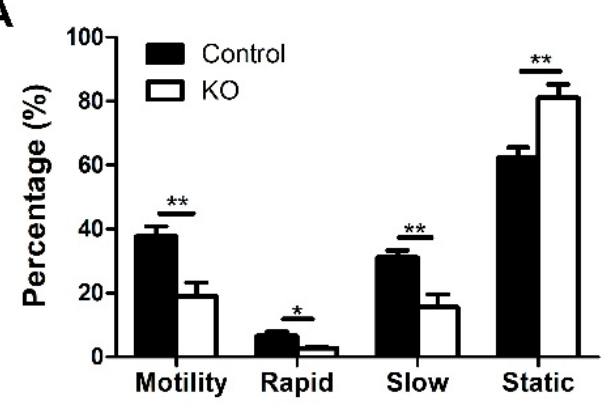

C

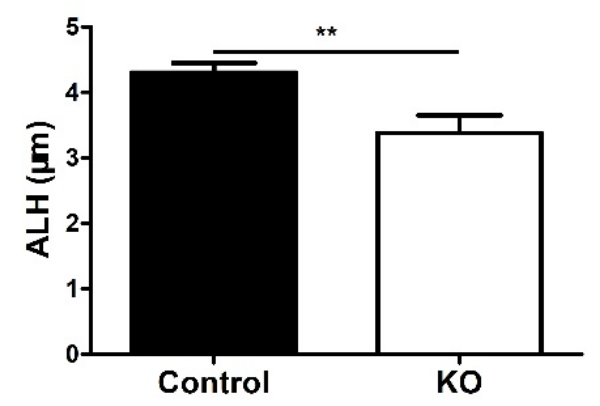

E Control
B

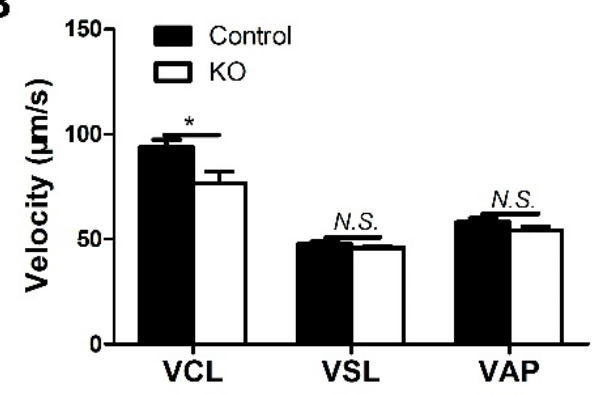

D

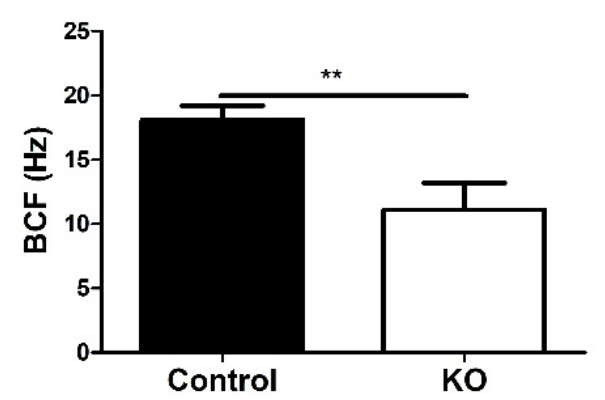

Zdhhc19-KO
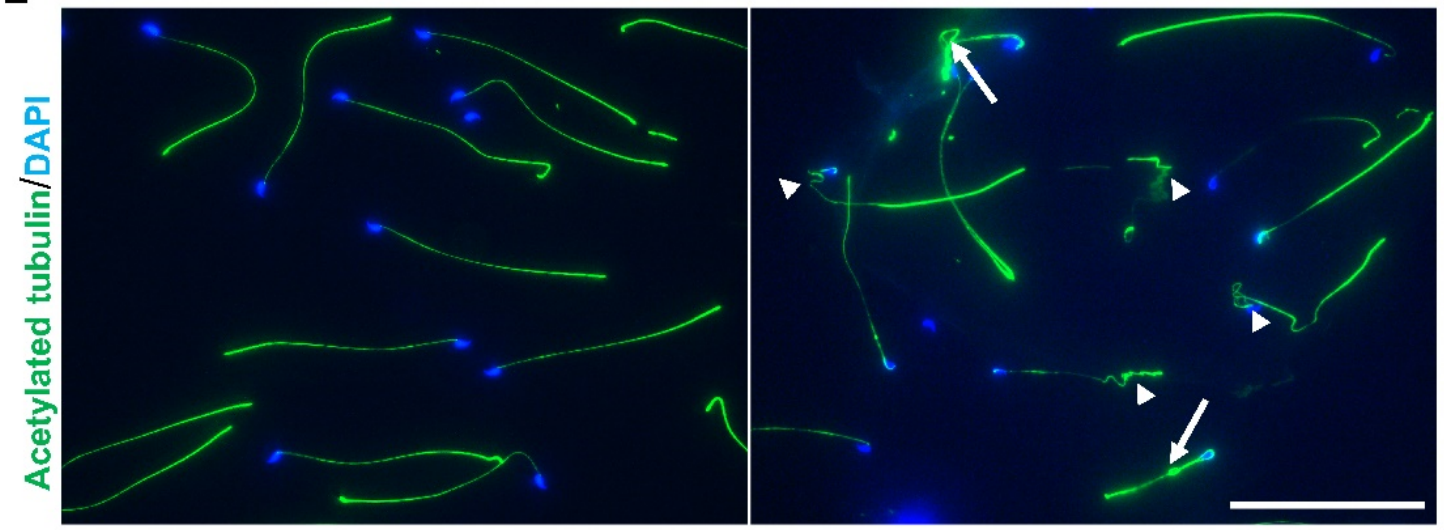

F

G
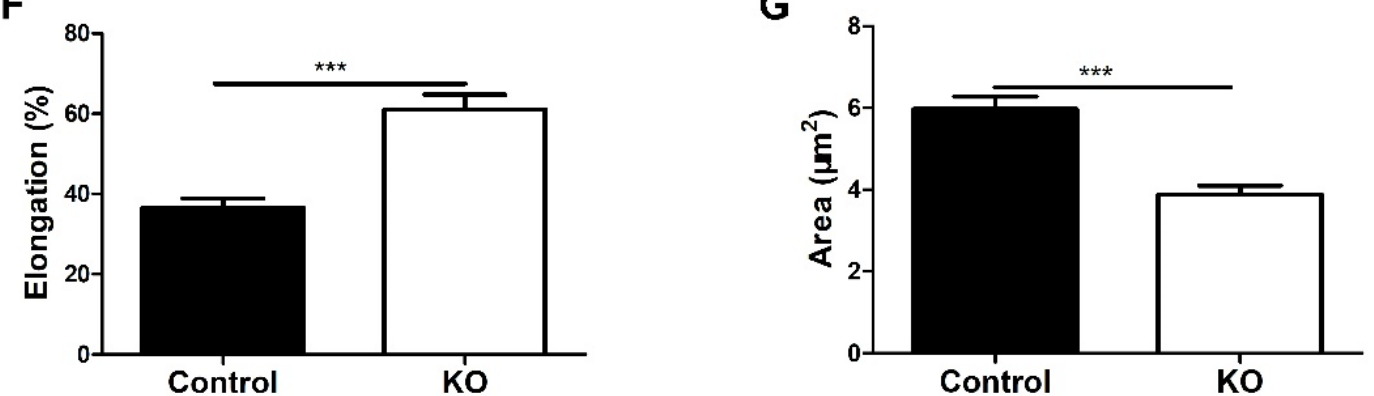

Figure 4. Zdhhc19 deletion caused abnormal sperm motility and sperm flagella. (A) CASA analyses of sperm motility on Zdhhc19 KO and control littermates. (B) Analyses of sperm velocity by CASA. VCL, curvilinear velocity; VSL, straight line velocity; VAP, average path velocity. (C) Analyses of sperm amplitude of lateral head displacement (ALH) by CASA. (D) Analyses of sperm beat/cross frequency (BCF) by CASA. (E) Immunofluorescence analyses of acetylated-TUBULIN on spermatozoa from adult KO and control littermates, counter-stained with DAPI. White arrows indicate sperm with bent tails, while arrowheads point to coiled sperm tails. Scale bars, $200 \mu \mathrm{m}$. $(\mathrm{F}, \mathrm{G})$ Analyses of sperm elongation $(\mathbf{F})$ and sperm area (G) by CASA. (A-D,F,G) Data are represented as the mean \pm SEM of three sperm samples per group. ${ }^{*} p<0.05$; ${ }^{* *} p<0.01,{ }^{* * *} p<0.001$; N.S.: no significance. 
To further understand whether the structure of sperm in Zdhhc19 KO mice was altered, we collected spermatozoa from cauda epididymides and conducted immunofluorescence (IF) analyses with an antibody against acetylated-TUBULIN, which recognize the antigen in sperm flagella. We found various morphological abnormalities in Zdhhc19 KO sperm tails, including coiled (arrowhead) and bent (arrow) sperm flagella, whereas sperm from control littermates developed normally (Figure 4E).

We then examined the structure of the sperm heads using IF assays with an acetylatedTUBULIN antibody and PNA staining. We did not find obvious abnormalities in heads of Zdhhc19 KO sperm (Supplementary Figure S1). However, CASA quantification revealed a higher width/length ratio (Elongation) and a smaller square of sperm head (Area) in Zdhhc19 KO sperm, compared with control sperm (Figure 4F,G), indicating that the loss of $Z d h h c 19$ also causes abnormalities in sperm head development. The exact abnormalities of sperm heads upon $Z d h h c 19 \mathrm{KO}$ may require ultrastructural analyses using transmission electron microscopy. Taken together, despite the fact that $Z d h h c 19$ is not required for spermatogenesis, Zdhhc19 deficiency causes structural abnormalities in sperm tails and probably in sperm heads as well.

\subsection{Zdhhc19 Is Dispensable for Acrosome Biogenesis but Is Required for the Acrosome Reaction}

The acrosome is a specialized organelle that covers the anterior part of the sperm head and contains hydrolytic enzymes that are essential for acrosome reaction to help sperm penetrate the zona pellucida (ZP) of oocytes. Therefore, properly formed acrosome and appropriate acrosome reaction play critical roles in mammalian fertilization [34]. Because Zdhhc19 KO mice are infertile with possible abnormalities in sperm heads, we sought to investigate whether $Z d h h c 19$ is required for acrosome biogenesis and proper acrosome reaction.

We performed immunostaining assays with Rhodamine-PNA (red). In this assay, intact acrosomes are labeled with Rhodamine-PNA, whereas sperm that had undergone acrosome reaction will be stained negative. As shown in Figure 5A, the acrosomes in sperm from both $\mathrm{KO}$ and control males were formed properly, as labeled with the red fluorescent Rhodamine-PNA dye (Figure 5A). About 18\% of sperm from Zdhhc19 KO mice underwent spontaneous acrosome reaction, similar to that of control littermates (Figure 5B). When treated with A23187, a commonly used chemical that induces the acrosome reaction in vitro, more than $40 \%$ of sperm from control mice responded well and underwent acrosome reaction (Figure 5A,B). By contrast, the ratio of Rhodamine-PNA negative sperm remained around $18 \%$ upon $Z$ dhhc19 deletion (Figure $5 \mathrm{~A}, \mathrm{~B}$ ). These results suggest that $Z$ dhhc19 is essential for induced acrosome reaction, but dispensable for acrosome biogenesis.

\subsection{Zdhhc19 Is Essential for In Vitro Fertilization in Mice}

Given that $Z d h h c 19 \mathrm{KO}$ mice were sterile yet with normal spermatogenesis, reduced sperm motility, and abnormal acrosome reaction, male infertility can be caused by failure of spermatozoa to migrate through the female uterotubal junction (UTJ) due to low sperm motility [35]. Alternatively, the sterility of Zdhhc19 KO mice may be attributed to defects in induced acrosome reaction and oocyte interaction/activation. To further distinguish the potential causes for male infertility upon $Z d h h c 19 \mathrm{KO}$, we performed the in vitro fertilization (IVF) assay, which can exclude the contribution of failure in UTJ migration of Zdhhc19 KO sperm. We used cumulus-intact eggs and monitored their fertilization by sperm to become two-cell embryos. Interestingly, we found a dramatically lower percentage (6/127 KO vs. 74/136 WT) of two-cell stage embryos after IVF with Zdhhc19 $\mathrm{KO}$ sperm, compared to that of wild type sperm (Figure 6A,B). 

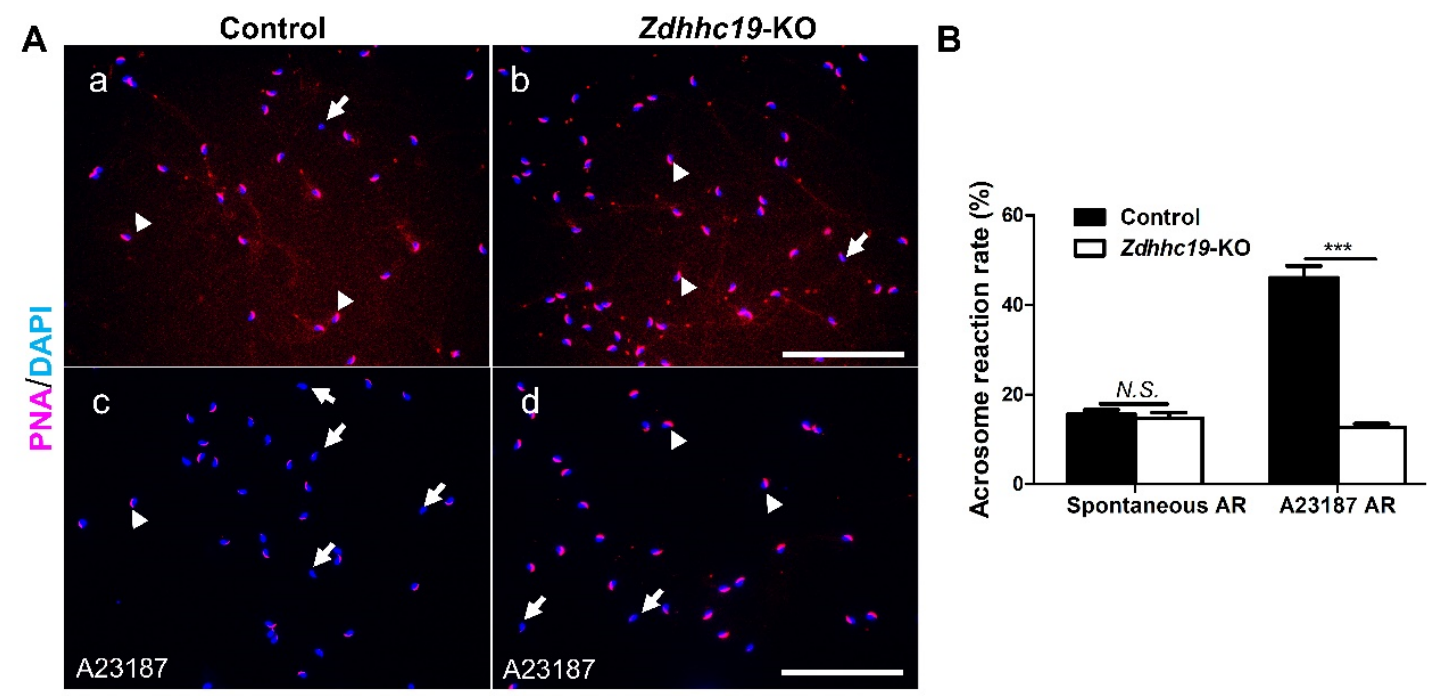

Figure 5. The induced acrosome reaction rate was significantly reduced upon Zdhhc19 KO. (A) Sperm without (a,b) or with treatment of A23187 (c,d) were stained with Rhodamine-PNA (red) and counter-stained with DAPI (blue). The acrosome-reacted sperm had DAPI-labeled nuclei but lacked PNA staining (white arrows), whereas intact acrosomes were stained positive for both PNA and DAPI-labeled nuclei (white arrow heads). Scale bars, $200 \mu \mathrm{m}$. (B) The proportions of sperm that underwent spontaneous AR and induced AR were calculated from between Zdhhc19 KO and control littermates. Data are represented as the mean \pm SEM of three sperm samples per group. N.S.: no significance; ${ }^{* * *} p<0.001$.

A

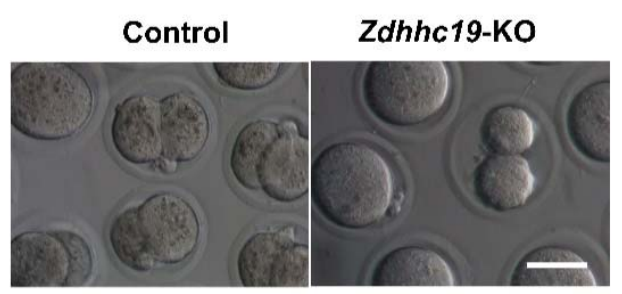

B

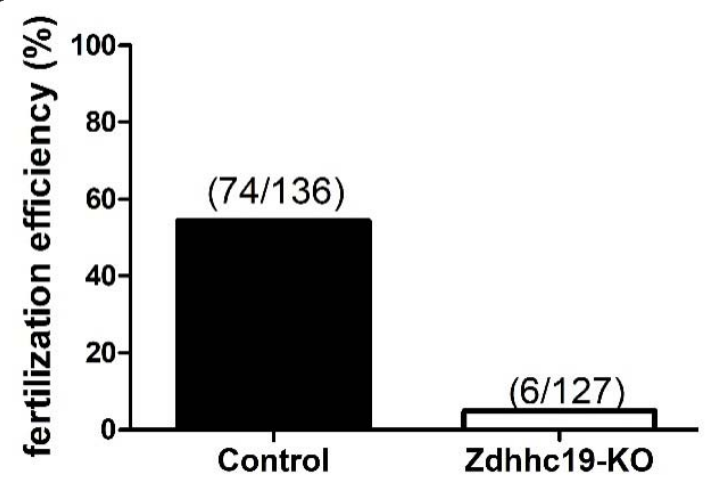

Figure 6. IVF analysis of Zdhhc19 KO spermatozoa. (A) Cumulus-intact eggs were incubated in vitro with capacitated Zdhhc19 KO or control sperm. Scale bar, $200 \mu \mathrm{m}$. (B) The percentage of 2-cell stage embryos upon IVF with control or KO sperm were calculated.

\section{Discussion}

Gene-expression analysis studies estimate that more than 2300 genes in the mouse genome are expressed predominantly in the male germ line [36]. These testis-enriched genes are thought to play important roles in spermatogenesis and fertilization [37]. Indeed, some testis specific genes were found to be necessary for spermatogenesis and male fertility, such as the testis expressed gene 14 (TEX14) [38], the oocyte fusion factor IZUMO1 [39], and the component of the sperm flagellar dynein regulatory complex TCTE1 [40]. However, Ikawa et al. reported about 100 evolutionarily conserved and testis enriched genes were dispensable for male mouse fertility $[37,41,42]$. Up to now, many testes specific genes remain unexplored for their exact roles in spermatogenesis and male reproduction. Gene knockout is the "gold standard" to determine the physiological roles of a gene in vivo. With the development of the CRISPR/Cas9 gene editing technique, it is now possible to rapidly generate a knockout mouse model in a cost-effective manner. In this study, we established Zdhhc19 KO mice by the CRISPR/Cas9 system to study its roles in spermatogenesis. 
Zdhhc19 is a testis-enriched gene that is highly conserved in all the eutherians from yeast to mammal [27]. The protein encoded by Zdhhc19 consists of zinc finger DHHC-Cys-rich domains which are the catalytic region for its palmitoyl $S$-acyltransferase activity [22,27]. The expression profile of Zdhhc19 in mouse tissues shows that $Z d h h c 19$ transcript is predominantly present in mouse testes (Mouse ENCODE transcriptome data: https:/ / www.ncbi.nlm.nih.gov/ gene/245308, updated on 23 June 2021). We also found Zdhhc19 mRNAs mainly in postnatal germ cells, suggesting that $Z d h h c 19$ plays potential roles in regulating spermatogenesis and/or fertility in mice. However, the specific types of germ cells (i.e., spermatogonia, spermatocyte, and haploid spermatid) in which ZDHHC19 is expressed in the testis are not known due to lack of commercial antibodies against this protein. To investigate the exact expression pattern of ZDHHC19 in the testis, specific antibodies for IHF should be developed. The transgenic reporter mice by knocking in a GFP cDNA under a Zdhhc19 promoter may also be used.

After successfully generating the $Z d h h c 19 \mathrm{KO}$ mouse model, we examined postnatal germ cell development upon loss of function of $Z d h h c 19$. We found that spermatocytes and spermatids developed normally in Zdhhc19 $\mathrm{KO}$ mice, which are however male infertile. One possibility of such phenomena may be due to the functional redundancy of other ZDHHC family members. For example, Zdhhc11, which is also expressed in mouse testis (our unpublished data), may work to compensate for the loss of function of Zdhhc19 in early spermatogenesis. Alternatively, we found that Zdhhc19 expression increased during spermatogenesis and peaked at postnatal week $4-5$ (Figure 1B), when elongating or elongated spermatids first appear in the testis [43]. Therefore, ZDHHC19 may have a pivotal role in forming functional spermatozoa/sperm in the epididymis, rather than spermatogenesis in the testis.

Sperm motility is highly related to their tail structures and functions of sperm tail proteins [44]. Indeed, our sperm morphology analysis showed that Zdhhc19 KO sperm had coiled and bent tails (Figure 4E). Under natural fertilization conditions, sperm must migrate through the UTJ, penetrate into the zona pellucida, and fuse to the oocyte [35]. Although spermatozoa with low motility may not reach UTJ, it is possible that they are capable of penetrating eggs in vitro. We thus performed IVF assay, and found that Zdhhc19 KO spermatozoa with low motility could not fertilize intact oocytes, indicating that the reduced sperm motility only partially accounts for the sterility in $\mathrm{KO}$ mice. Because Zdhhc19 KO spermatozoa contained potential defects in their head formation (Figure $4 \mathrm{~F}, \mathrm{G}$ ) and did not respond well to A23187 (Figure 5A,B), it is plausible that impaired ability of Zdhhc19 KO sperm in induced acrosome reaction is a primary cause for their male infertility.

The exact molecular mechanism by which ZDHHC19 regulates sperm functions remains unknown. Palmitoylation has been shown to be required for protein stability, localization, enzymatic activities, and protein-protein and protein-lipid interactions $[27,29,45]$. As a palmitoyl S-acyltransferase, ZDHHC19 likely acts through its substrates to regulate sperm functions. Currently, the protein targets of ZDHHC19 in germ cells are yet to be discovered. Given that the peak expression of ZDHHC19 occurs in the late stage of spermatogenesis, we speculate that ZDHHC19 acts upon de novo synthesized motor proteins and/or structure-related cytoskeleton proteins for palmitoylation during epididymal maturation, which in turn impact acrosome reaction, sperm tail/head development, and sperm mobility. Future studies are warranted to identify the specific palmitoylated substrates by ZDHHC19 to further elucidate its molecular mechanism in sustaining sperm functions.

\section{Materials and Methods}

\subsection{Mouse Lines, Animal Care, and Fertility Test}

Zdhhc19 knockout mice were generated in Cyagen Biosciences (Suzhou, China) using the CRISPR/Cas9 techniques. The strategy for gene targeting is shown in Figure 2A. The target DNA sequences with PAM were: gRNA-1-AATGCGCAGCCATAGAATCCTGG and gRNA-2-GTGTGGTCCACGGTGTTAGCAGG. The sequence primer was: GTGTTTGCTGCCTTCAATGTAACG. For the generation of Zdhhc19 KO mice, the female and male founders carrying a heterozygous $(+/-)$ deletion mutation were inbred to produce ho- 
mozygous ( $-/-)$ mice. The progeny mice were genotyped with PCR. For the fertility test, three adult $\mathrm{KO}$ and control males were separately paired with two sexually mature wild-type females for at least 2 months. The number of pups were counted and recorded. Pups per litter were presented as the number of total pups born divided by the number of litters. All animal experimental procedures (Protocol ID: AR201305007) were conducted in accordance with the local Animal Welfare Act and Public Health Service Policy (consistent with the WMA Statement on Animal Use in Biomedical Research) and approved on 1 May 2013 by the Committee of Animal Experimental Ethics at East China Normal University.

\subsection{Genomic DNA Extraction and Genotyping}

Genomic DNA was isolated as described previously [46]. Briefly, tail tips were digested in buffer A at $95^{\circ} \mathrm{C}$ for $30 \mathrm{~min}$, and the reactions were then stopped by adding buffer B. The supernatant was collected for PCR genotyping with Taq Master Mix (Vazyme Biotech, Nanjing, China, P112-AA) according to the manufacturer's instructions. Sequences of primers and size of products are provided in Supplementary Table S1.

\subsection{Total RNA Extraction, Reverse Transcription, and Real-Time PCR}

As previously described [46], total RNA was extracted with RNAiso Plus solution (TAKARA, Dalian, China, 9109), and cDNAs were synthesized using a PrimeScrip RT reagent Kit (TAKARA, RR037A). Quantitative Real-time PCR was performed on Thermo Scientific QuantStudio 3 Real-Time PCR System using FastStart Universal SYBR Green Master (Roche Life Science, Mannheim, Germany, 04913914001). The data were analyzed using the comparative threshold cycle $(\Delta \Delta \mathrm{Ct})$ method and normalized to Gapdh. All PCR primers used are listed in Supplementary Table S2.

\subsection{Histology and Immunohistofluorensce (IHF)}

IHF was performed as described previously [46]. Testes were fixed in $4 \%$ paraformaldehyde (PFA) at $4{ }^{\circ} \mathrm{C}$ overnight and embedded in paraffin. Antibodies used in this study, TRA98 (ab82527; 1:500), SYCP3 (ab15093; 1:400), and TNP1 (ab73135; 1:1000), were purchased from Abcam (Cambridge, MA, USA). Alexa Fluor ${ }^{\circledR}$ conjugated secondary antibodies came from Jackson ImmunoResearch Laboratories (West Grove, PA, USA). Nuclei were counterstained with DAPI/ProLong ${ }^{\mathrm{TM}}$ Diamond Antifade Mountant (Thermo Fisher Scientific, Eugene, OR, USA, P36966). All images were collected using an Olympus BX53 microscope system (Olympus Life Science, Japan), and Brightness/Contrast and Channel Merges of pictures were processed with Image J software.

\subsection{Immunofluorescence Staining (IF)}

Sperm was collected after swimming out from the cauda epididymides into1x PBS, then were mounted on glass slides and dried. Slides were fixed in $4 \%$ paraformaldehyde (PFA) and permeabilized with $0.2 \%$ Triton X-100. After washing with PBS, slides were blocked by $10 \%$ Fetal Bovine Serum (FBS) (in $1 \times$ PBS with $0.5 \%$ Tween-20) at room temperature for $1 \mathrm{~h}$, followed with primary antibodies incubation at $4{ }^{\circ} \mathrm{C}$ overnight, PBST ( $1 \times$ PBS with $0.1 \%$ Tween 20) washed sperm sections for 5 min thrice, incubated with secondary antibodies (in $1 \times$ PBS with $1 \%$ BSA) for $1 \mathrm{~h}$ at room temperature, counterstained with DAPI/ProLong ${ }^{\mathrm{TM}}$ Diamond Antifade Mountant. Immunofluorescence Images were captured and observed by Olympus BX53 microscope system, and processed with Image J. An antibody in this study, Acetylated TUBULIN (ab179484; 1:500) was purchased from Abcam. Alexa Fluor ${ }^{\circledR}$ conjugated secondary antibodies came from Jackson ImmunoResearch Laboratories. 


\subsection{Sperm Motility Analysis}

Sperm motility analysis was performed following a published protocol with minor modification [47]. Briefly, three $\mathrm{KO}$ and control male mice (12-16 weeks old) were sacrificed by cervical dislocation and sperm from the epididymis were collected and dispersed in $1 \mathrm{~mL}$ of $1 \times$ PBS. After incubation for $20 \mathrm{~min}$ at $37^{\circ} \mathrm{C}$ in air, a $20-\mu \mathrm{L}$ aliquot of the sperm suspension was then placed in a counting chamber (Leja SC100-01-02-A-CE) for assessment of motility by using an HTM-TOX IVOS sperm motility analyzer (version 14, Hamilton Thorne Biosciences). The parameters assayed were the percentage of motile sperm cells, Average Path Velocity (VAP), Straight-line Velocity (VSL), Curvilinear Velocity (VCL), Amplitude of Lateral Head displacement (ALH), and Beat Cross Frequency (BCF).

\subsection{In Vitro Fertilization in Mice (IVF)}

In vitro fertilization was performed as previously described with minor modifications [34]. Mature C57/B6L female mice were injected with pregnant mare serum gonadotropin (PMSG) (Ningbo Sansheng Pharmaceutical Co., Ltd., Ningbo, China) followed by Human Chorionic Gonadotropin (hCG) (Ningbo Sansheng Pharmaceutical co., Ltd., China) after $48 \mathrm{~h}$. The female mice were then euthanized by cervical dislocation, after injecting hCG $13 \mathrm{~h}$. Oviducts were collected in a 35-mm dish containing HTF medium (Nanjing Aibei Biotechnology Co., Ltd., Nanjing, China, M1130), and stage MII oocytes were collected from the oviducts. Mature sperm were collected from the cauda epididymis and transferred into the HTF medium for capacitation at $37{ }^{\circ} \mathrm{C}$ in a humidified incubator with $5 \% \mathrm{CO}_{2}, 95 \%$ air. After 30-60 min, the capacitated sperm were added to the fertilization droplet containing the oocytes. After 4-6 h of incubation, the oocytes were transferred to KSOM medium. The IVF rate was measured based on the proportion of 2-cell embryos at $24 \mathrm{~h}$ after insemination.

\subsection{Acrosome Reaction Analysis}

Mature sperm from the cauda epididymis were incubated in HTF medium at $37^{\circ} \mathrm{C}$ in a humidified incubator with 5\% CO2 and 95\% air to allow capacitation. After $1 \mathrm{~h}$, the calcium ionophore A23187 (MedChemExpressm LLC, Shanghai, China, HY-N6687) was added at final concentration of $20 \mu \mathrm{m}$ to induce the acrosome reaction. After an additional $1 \mathrm{~h}$ incubation, sperm were smeared on a glass microscope slide, dried at room temperature, and fixed with methanol at $20^{\circ} \mathrm{C}$ for $30 \mathrm{~s}$. Intact acrosomes were stained with Rhodamine-PNA (Vector Laboratories, RL-1072; 1:1000), and the sperm nuclei were labeled with DAPI. The acrosome status was evaluated by staining with Rhodamine-PNA, which binds the outer acrosomal membrane and therefore would not stain acrosome-damaged and acrosome-reacted sperm. More than 200 sperm were examined for all experimental conditions [48].

\subsection{Statistics}

The data were evaluated for significant differences using Student's $t$-test and the 2-way ANOVA, calculated with GraphPadPrism5 software (GraphPad Software, La Jolla, CA, USA). A $p$-value $<0.05$ was considered statistically significant.

Supplementary Materials: Supplementary materials can be found at https://www.mdpi.com/ article/10.3390/ijms22168894/s1.

Author Contributions: S.W. and D.Q. designed research, S.W. performed experiments and wrote manuscript, S.W. analyzed the data, P.W. contributed to the mouse keeping, H.Q. contributed with the methodology and D.Q. and Y.W. revised the manuscript. All authors have read and agreed to the published version of the manuscript.

Funding: This work was supported by grants from the Natural Science Foundation of Guangdong Province of China (14162144) and the Li Ka Shing Foundation Postdoctoral Research Fellowship Program at Shantou University Medical College (LD0102/510859030). Yuan Wang is partially supported by NIFA through AgbioResearch Hatch Fund at Michigan State University. 
Institutional Review Board Statement: The study was conducted according to the guidelines of the Declaration of Helsinki, and approved by the Ethics Committee of East China Normal University (protocol code AR201305007) on 1 May 2013.

Informed Consent Statement: Not applicable.

Data Availability Statement: The data presented in this study are available on request from the corresponding author.

Acknowledgments: We would like to thank Hongjie Qiao, Ni Zhang, Li Hu, and all members of the Wang lab for comments and scientific input. We thank Yuan Wang for providing the necessary facilities to perform experiments. We also thank Tengfei Liu (Laboratory Animal Research Center, East China Normal University) for technical support in IVF, Weiwei Wang (Shanghai Institute of Planned Parenthood Research) for CASA service, and Shuiping Li (Shanghai Jiao Tong University School of Medicine) for image processing.

Conflicts of Interest: The authors declare no conflict of interest.

\section{References}

1. Russell, L.D.; Ettlin, R.A.; Hikim, A.P.S.; Clegg, E.D. Histological and histopathological evaluation of the testis. Int. J. Androl. 1993, 16, 83. [CrossRef]

2. Sun, G.; Jiang, M.; Zhou, T.; Guo, Y.; Cui, Y.; Guo, X.; Sha, J. Insights into the lysine acetylproteome of human sperm. J. Proteom. 2014, 109, 199-211. [CrossRef] [PubMed]

3. Yu, H.; Diao, H.; Wang, C.; Lin, Y.; Yu, F.; Lu, H.; Xu, W.; Li, Z.; Shi, H.; Zhao, S.; et al. Acetylproteomic analysis reveals functional implications of lysine acetylation in human spermatozoa (sperm). Mol. Cell. Proteom. 2015, 14, 1009-1023. [CrossRef]

4. Tan, M.; Luo, H.; Lee, S.; Jin, F.; Yang, J.S.; Montellier, E.; Buchou, T.; Cheng, Z.; Rousseaux, S.; Rajagopal, N.; et al. Identification of 67 histone marks and histone lysine crotonylation as a new type of histone modification. Cell 2011, 146, 1016-1028. [CrossRef]

5. Wang, G.; Wu, Y.; Zhou, T.; Guo, Y.; Zheng, B.; Wang, J.; Bi, Y.; Liu, F.; Zhou, Z.; Guo, X.; et al. Mapping of the N-linked glycoproteome of human spermatozoa. J. Proteome Res. 2013, 12, 5750-5759. [CrossRef] [PubMed]

6. Yang, X.; Liu, F.; Yan, Y.; Zhou, T.; Guo, Y.; Sun, G.; Zhou, Z.; Zhang, W.; Guo, X.; Sha, J. Proteomic analysis of N-glycosylation of human seminal plasma. Proteomics 2014, 15, 1255-1258. [CrossRef] [PubMed]

7. Lan, R.; Xin, M.; Hao, Z.; You, S.; Xu, Y.; Wu, J.; Dang, L.; Zhang, X.; Sun, S. Biological functions and large-scale profiling of protein glycosylation in human semen. J. Proteome Res. 2020, 19, 3877-3889. [CrossRef] [PubMed]

8. Qi, L.; Liu, Z.; Wang, J.; Cui, Y.; Guo, Y.; Zhou, T.; Zhou, Z.; Guo, X.; Xue, Y.; Sha, J. Systematic analysis of the phosphoproteome and kinase-substrate networks in the mouse testis. Mol. Cell. Proteom. 2014, 13, 3626-3638. [CrossRef]

9. Castillo, J.; Knol, J.C.; Korver, C.M.; Piersma, S.R.; Pham, T.V.; Haas, R.R.D.G.-D.; van Pelt, A.; Jimenez, C.R.; Jansen, B.J.H. Human testis phosphoproteome reveals kinases as potential targets in spermatogenesis and testicular cancer. Mol. Cell. Proteom. 2019, 18, S132-S144. [CrossRef]

10. Li, Y.; Cheng, Y.; Zhu, T.; Zhang, H.; Li, W.; Guo, Y.; Qi, Y.; Chen, X.; Zhang, J.; Sha, J.; et al. The protein phosphorylation landscape of mouse spermatids during spermiogenesis. Proteomics 2019, 19, e1900055. [CrossRef]

11. Cai, L.; Tu, J.; Song, L.; Gao, Z.; Li, K.; Wang, Y.; Liu, Y.; Zhong, F.; Ge, R.; Qin, J.; et al. Proteome-wide mapping of endogenous sumoylation sites in mouse testis. Mol. Cell. Proteom. 2017, 16, 717-727. [CrossRef]

12. Hou, X.; Zhang, W.; Xiao, Z.; Gan, H.; Lin, X.; Liao, S.; Shangying, L. Mining and characterization of ubiquitin E3 ligases expressed in the mouse testis. BMC Genom. 2012, 13, 495. [CrossRef]

13. MacLeod, G.; Varmuza, S. The application of proteomic approaches to the study of mammalian spermatogenesis and sperm function. FEBS J. 2013, 280, 5635-5651. [CrossRef] [PubMed]

14. Brohi, R.D.; Huo, L.-J. Posttranslational modifications in spermatozoa and effects on male fertility and sperm viability. OMICS J. Integr. Biol. 2017, 21, 245-256. [CrossRef] [PubMed]

15. Bhagwat, S.; Dalvi, V.; Chandrasekhar, D.; Matthew, T.; Acharya, K.; Gajbhiye, R.; Kulkarni, V.; Sonawane, S.; Ghosalkar, M.; Parte, P. Acetylated $\alpha$-tubulin is reduced in individuals with poor sperm motility. Fertil. Steril. 2014, 101, 95-104.e3. [CrossRef]

16. Shumeng, L.; Huajing, Y.; Yongqing, L.; Xinhua, L.; Yu, Z.; Chen, B.; Shuai, Y.; Zhe, C.; Guojia, X.; Wanjin, L.; et al. Chromodomain protein cdyl acts as a crotonyl-coa hydratase to regulate histone crotonylation and spermatogenesis. Mol. Cell 2017, 67, 853-866.

17. Fukuda, M.N.; Akama, O.T. In vivo role of $\alpha$-mannosidase IIx: Ineffective spermatogenesis resulting from targeted disruption of the Man2a2 in the mouse. Biochim. Biophys. Acta (BBA) Gen. Subj. 2002, 1573, 382-387. [CrossRef]

18. Li, Y.; Liu, W.-S.; Yi, J.; Kong, S.-B.; Ding, J.-C.; Zhao, Y.-N.; Tian, Y.-P.; Feng, G.-S.; Li, C.-J.; Liu, W.; et al. The role of tyrosine phosphatase Shp2 in spermatogonial differentiation and spermatocyte meiosis. Asian J. Androl. 2020, 22, 79-87. [CrossRef] [PubMed]

19. Gou, L.-T.; Kang, J.-Y.; Dai, P.; Wang, X.; Li, F.; Zhao, S.; Zhang, M.; Hua, M.-M.; Lu, Y.; Zhu, Y.; et al. Ubiquitination-deficient mutations in human piwi cause male infertility by impairing histone-to-protamine exchange during spermiogenesis. Cell 2017, 169, 1090-1104.e13. [CrossRef] 
20. Smotrys, J.E.; Linder, M.E. Palmitoylation of intracellular signaling proteins: Regulation and function. Annu. Rev. Biochem. 2004, 73, 559-587. [CrossRef]

21. Jiang, H.; Zhang, X.; Chen, X.; Aramsangtienchai, P.; Tong, Z.; Lin, H. Protein lipidation: Occurrence, mechanisms, biological functions, and enabling technologies. Chem. Rev. 2018, 118, 919-988. [CrossRef] [PubMed]

22. Ko, P.; Dixon, S.J. Protein palmitoylation and cancer. EMBO Rep. 2018, 19, e46666. [CrossRef]

23. Fukata, M.; Fukata, Y.; Adesnik, H.; Nicoll, R.A.; Bredt, D.S. Identification of PSD-95 palmitoylating enzymes. Neuron 2004, 44, 987-996. [CrossRef]

24. Linder, M.E.; Jennings, B.C. Mechanism and function of DHHC S-acyltransferases. Biochem. Soc. Trans. 2013, 41, 29-34. [CrossRef] [PubMed]

25. Rocks, O.; Gerauer, M.; Vartak, N.; Koch, S.; Huang, Z.-P.; Pechlivanis, M.; Kuhlmann, J.; Brunsveld, L.; Chandra, A.; Ellinger, B.; et al. The palmitoylation machinery is a spatially organizing system for peripheral membrane proteins. Cell 2010, 141, 458-471. [CrossRef]

26. Gorleku, O.A.; Barns, A.-M.; Prescott, G.R.; Greaves, J.; Chamberlain, L.H. Endoplasmic reticulum localization of DHHC palmitoyltransferases mediated by lysine-based sorting signals. J. Biol. Chem. 2011, 286, 39573-39584. [CrossRef]

27. Ohno, Y.; Kihara, A.; Sano, T.; Igarashi, Y. Intracellular localization and tissue-specific distribution of human and yeast DHHC cysteine-rich domain-containing proteins. Biochim. Biophys. Acta (BBA) Mol. Cell Biol. Lipids 2006, 1761, 474-483. [CrossRef]

28. Lanyon-Hogg, T.; Faronato, M.; Serwa, R.; Tate, E.W. Dynamic protein acylation: New substrates, mechanisms, and drug targets. Trends Biochem. Sci. 2017, 42, 566-581. [CrossRef] [PubMed]

29. Resh, M.D. Fatty acylation of proteins: New insights into membrane targeting of myristoylated and palmitoylated proteins. Biochim. Biophys. Acta (BBA) Bioenerg. 1999, 1451, 1-16. [CrossRef]

30. Liu, J.-G.; Yuan, L.; Brundell, E.; Bjorkroth, B.; Daneholt, B.; Höög, C. Localization of the N-terminus of SCP1 to the central element of the synaptonemal complex and evidence for direct interactions between the N-termini of SCP1 molecules organized head-to-head. Exp. Cell Res. 1996, 226, 11-19. [CrossRef]

31. Tanaka, H.; Pereira, L.A.V.D.; Nozaki, M.; Tsuchida, J.; Sawada, K.; Mori, H.; Nishimune, Y. A germ cell-specific nuclear antigen recognized by a monoclonal antibody raised against mouse testicular germ cells. Int. J. Androl. 1998, 20, 361-366. [CrossRef] [PubMed]

32. Heidaran, M.A.; Showman, R.M.; Kistler, W.S. A cytochemical study of the transcriptional and translational regulation of nuclear transition protein 1 (TP1), a major chromosomal protein of mammalian spermatids. J. Cell Biol. 1988, 106, 1427-1433. [CrossRef]

33. Orgebin-Crist, M.C. Sperm maturation in rabbit epididymis. Nat. Cell Biol. 1967, 216, 816-818. [CrossRef]

34. Hao, J.; Chen, M.; Ji, S.; Wang, X.; Wang, Y.; Huang, X.; Yang, L.; Wang, Y.; Cui, X.; Lv, L.; et al. Equatorin is not essential for acrosome biogenesis but is required for the acrosome reaction. Biochem. Biophys. Res. Commun. 2014, 444, 537-542. [CrossRef] [PubMed]

35. Larasati, T.; Noda, T.; Fujihara, Y.; Shimada, K.; Tobita, T.; Yu, Z.; Matzuk, M.M.; Ikawa, M. Tmprss12 is required for sperm motility and uterotubal junction migration in mice. Biol. Reprod. 2020, 103, 254-263. [CrossRef] [PubMed]

36. Schultz, N.; Hamra, F.K.; Garbers, D.L. A multitude of genes expressed solely in meiotic or postmeiotic spermatogenic cells offers a myriad of contraceptive targets. Proc. Natl. Acad. Sci. USA 2003, 100, 12201-12206. [CrossRef]

37. Miyata, H.; Castaneda, J.M.; Fujihara, Y.; Yu, Z.; Archambeault, D.R.; Isotani, A.; Kiyozumi, D.; Kriseman, M.L.; Mashiko, D.; Matsumura, T.; et al. Genome engineering uncovers 54 evolutionarily conserved and testis-enriched genes that are not required for male fertility in mice. Proc. Natl. Acad. Sci. USA 2016, 113, 7704-7710. [CrossRef]

38. Greenbaum, M.P.; Yan, W.; Wu, M.-H.; Lin, Y.-N.; Agno, J.E.; Sharma, M.; Braun, R.E.; Rajkovic, A.; Matzuk, M.M. TEX14 is essential for intercellular bridges and fertility in male mice. Proc. Natl. Acad. Sci. USA 2006, 103, 4982-4987. [CrossRef] [PubMed]

39. Inoue, N.; Ikawa, M.; Isotani, A.; Okabe, M. The immunoglobulin superfamily protein Izumo is required for sperm to fuse with eggs. Nat. Cell Biol. 2005, 434, 234-238. [CrossRef]

40. Castaneda, J.M.; Hua, R.; Miyata, H.; Oji, A.; Guo, Y.; Cheng, Y.; Zhou, T.; Guo, X.; Cui, Y.; Shen, B.; et al. TCTE1 is a conserved component of the dynein regulatory complex and is required for motility and metabolism in mouse spermatozoa. Proc. Natl. Acad. Sci. USA 2017, 114, E5370-E5378. [CrossRef]

41. Lu, Y.; Oura, S.; Matsumura, T.; Oji, A.; Sakurai, N.; Fujihara, Y.; Shimada, K.; Miyata, H.; Tobita, T.; Noda, T.; et al. CRISPR/Cas9mediated genome editing reveals 30 testis-enriched genes dispensable for male fertility in micet. Biol. Reprod. 2019, 101, 501-511. [CrossRef] [PubMed]

42. Park, S.; Shimada, K.; Fujihara, Y.; Xu, Z.; Shimada, K.; Larasati, T.; Pratiwi, P.; Matzuk, R.M.; Devlin, D.J.; Yu, Z.; et al. CRISPR/Cas9-mediated genome-edited mice reveal 10 testis-enriched genes are dispensable for male fecundity. Biol. Reprod. 2020, 103, 195-204. [CrossRef] [PubMed]

43. Drumond-Bock, A.L.; Meistrich, M.L.; Chiarini-Garcia, H. Spermatogonial morphology and kinetics during testis development in mice: A high-resolution light microscopy approach. Reproduction 2011, 142, 145-155. [CrossRef] [PubMed]

44. Lehti, M.S.; Sironen, A. Formation and function of sperm tail structures in association with sperm motility defects. Biol. Reprod. 2017, 97, 522-536. [CrossRef] [PubMed]

45. Dunphy, J.T.; Linder, M.E. Signalling functions of protein palmitoylation. Biochim. Biophys. Acta (BBA) Mol. Cell Biol. Lipids 1998, 1436, 245-261. [CrossRef] 
46. Wang, S.; Wang, P.; Liang, D.; Wang, Y. BRG1 is dispensable for sertoli cell development and functions in mice. Int. J. Mol. Sci. 2020, 21, 4358. [CrossRef]

47. Zhou, Y.; Zheng, M.; Shi, Q.; Zhang, L.; Zhen, W.; Chen, W.; Zhang, Y. An epididymis-specific secretory protein HongrES1 critically regulates sperm capacitation and male fertility. PLoS ONE 2008, 3, e4106. [CrossRef]

48. Kitamura, K.; Tanaka, H.; Nishimune, Y.; Haprin, A. Novel haploid germ cell-specific RING finger protein involved in the acrosome reaction. J. Biol. Chem. 2003, 278, 44417-44423. [CrossRef] 